\title{
Erratum to: Uncertain Rule-Based Fuzzy Systems
}

\section{Erratum to: \\ J. M. Mendel, Uncertain Rule-Based Fuzzy Systems, https://doi.org/10.1007/978-3-319-51370-6}

In the original version of the book, extra server material has been included, extra's online logo has been added in the cover, and extra's link has been provided in the copyright page. The erratum book has been updated with the changes.

The updated online version of this book can be found at

https://doi.org/10.1007/978-3-319-51370-6 